Chirurg 2014 · 85:65

DOI 10.1007/s00104-013-2674-x

Online publiziert: 18. Dezember 2013

c) Springer-Verlag Berlin Heidelberg 2013
O. Strobel · M.W. Büchler

Klinik für Allgemein-, Viszeral- und Transplantationschirurgie, Universität Heidelberg

\section{Mesohepatektomie bei zentralen Lebertumoren}

\section{Originalpublikation}

Qiu J, Wu H, Bai Y et al (2013) Mesohepatectomy for centrally located liver tumours. Br J Surg 100:1620-1626

\section{Hintergrund und Fragestellung}

Bei zentralen Lebertumoren (CouinaudSegmente IV, V, VIII \pm I) werden meist erweiterte rechte oder linke Hemihepatektomien $(\mathrm{EH})$ durchgeführt. Durch den hohen Verlust von Leberparenchym sind diese Eingriffe mit einem hohen Risiko für ein postoperatives Leberversagen und weitere Komplikationen assoziiert und bei vorbestehendem Parenchymschaden oft nicht durchführbar. Zentrale Leberresektionen (Mesohepatektomien, $\mathrm{MH}$ ) erlauben durch eine isolierte Entfernung zentraler Lebersegmente einen maximalen Parenchym- und Funktionserhalt, sind aber technisch oft anspruchsvoller und aufgrund der zweifachen Transsektion theoretisch mit einem höheren Risiko für oberflächenassoziierte Komplikationen (Galleleck, Hämatom) assoziiert.

Qiu et al. verglichen in einer retrospektiven Studie in 471 Patienten die perioperativen Ergebnisse nach $\mathrm{MH}$ und $\mathrm{EH}$ bei zentralen Lebertumoren.

\section{Methoden}

Von 2005 bis 2011 wurden bei 491 konsekutiven Patienten zentrale Lebertumoren reseziert. Eine kompensierte Leberfunktion, die Möglichkeit der Major-Leberresektion und der En-bloc-Tumorresektion waren Einschlusskriterien. Die zusätzli- che Anwendung ablativer Verfahren war ein Ausschlussgrund. Es wurden die perioperativen Ergebnisse von 292 Patienten mit $\mathrm{MH}$ und 138 Patienten mit EH analysiert. Bei 89,1\% der Patienten lag ein hepatozelluläres Karzinom vor. Die Entscheidung für $\mathrm{MH}$ oder $\mathrm{EH}$ wurde intraoperativ individuell getroffen. Dabei wurden parenchymsparende Resektionen favorisiert: Es kamen verschiedene zentrale Leberresektionen (Segmente IVb + V, Segmente IVa + VIII oder Segmente Iva + Ivb + V + VIII) zur Anwendung und falls diese nicht möglich waren eine EH. Nach der Parenchymdurchtrennung mittels Ultraschall- oder Wasserdissektor wurde zur Lokalisation von Gallelecks eine intraoperative Cholangiographie durchgeführt. In Abhängigkeit der Lagebeziehung der Tumoren zu den Pedikelstrukturen und den Lebervenen erfolgte eine Klassifikation der durchgeführten $\mathrm{MH}$ in vier Typen zunehmender Komplexität.

\section{Ergebnisse}

Das Vorliegen einer Zirrhose war bei MH häufiger als bei $\mathrm{EH}$ (72,3 vs. $61,6 \%)$. Vor $\mathrm{EH}$ wurden häufiger Pfortaderembolisationen durchgeführt (10,1 vs. $2,4 \%)$. Ansonsten waren die Gruppen hinsichtlich präoperativer Parameter vergleichbar. MH dauerten signifikant länger als EH (259 vs. $211 \mathrm{~min}$ ). Bluttransfusionen waren bei $\mathrm{MH}$ signifikant häufiger (33,9 vs. $24,6 \%$ ). Die Komplikationsrate war nach $\mathrm{MH}$ signifikant niedriger als nach EH (19,9 vs. 52,2\%). Aszites, Pleuraergüsse, prolongierter Ikterus und Hyperbilirubinämie traten jeweils nach
EH signifikant häufiger auf als nach $\mathrm{MH}$. Die Rate an Gallelecks (2,7 vs. 0,7\%) und Hämatomen (1,0 vs. $1,4 \%)$ sowie die Mortalität $(0,7$ vs. $1,4 \%)$ waren vergleichbar. Bei den MH hatten Typ-IV-Eingriffe die längste Dauer, den größten Blutverlust, die höchste Transfusionsrate und die höchste Morbidität.

\section{Diskussion}

Die Studie zeigt, dass die MH eine sichere Alternative zur EH bei selektionierten Patienten ist. Allerdings sind die Gruppen $\mathrm{MH}$ und $\mathrm{EH}$ in der vorliegenden Studie aufgrund der Entscheidungsfindung hinsichtlich der Resektionsformen und des retrospektiven Designs nur eingeschränkt vergleichbar. Anhand der Literatur wird die Morbidität nach $\mathrm{MH}$ maßgeblich durch die Rate an Gallelecks beeinflusst, die bei Qiu et al. erstaunlich niedrig ist. Diese guten Ergebnisse nach $\mathrm{MH}$ sind wahrscheinlich nicht allgemein übertragbar. Die erhöhte Komplikationsrate nach den komplexen (Typ-IV-)MH zeigen, dass diese Eingriffe wie EH an Zentren durchgeführt werden sollten.

\section{Korrespondenzadresse}

\section{PD Dr. O. Strobel}

Klinik für Allgemein-, Viszeralund Transplantationschirurgie, Universität Heidelberg,

Im Neuenheimer Feld 110, 69120 Heidelberg

Oliver.Strobel@med.uni-heidelberg.de

Interessenkonflikt. O. Strobel und M.W. Büchler geben an, dass kein Interessenkonflikt besteht. 